\title{
PARALLELISM AND COMPLEXITY OF A SMALL-WORLD NETWORK MODEL
}

\author{
Robert E. Hiromoto \\ University of Idaho \\ Center for Advanced Energy Studies \\ Idaho Falls, Idaho, USA, hiromoto@uidaho.edu
}

\begin{abstract}
The small-world phenomena exhibits highly localized clustering and short-cut paths between vertices in a graph that reflect observed properties in social networks, epidemiological models and other real-world networks. The small-world models rely on the application of constraint-based randomness or the derivation of constraints on randomness to simulate the desired network complexities and their associated network connection properties. In this paper, rather than exploring the random properties of small-world networks, we employ deterministic strategies in the design of a computationally efficient distributed neuronal-axon network simulator that results in a small world network. These strategies are derived by addressing the parallel complexities of the proposed neuronal-axon network simulator, and also from physical constraints imposed by resource limitations of the distributed simulation architecture. The outcome of this study is the realization of a neuronal-axon network simulator that exhibits small-world characteristics of clustering with a logarithmic degree of separation between nodes without the need for long-range communication edges. The importance of this result is the deterministic application of reasoned optimization rules from which the small-world network emerges. Copyright (C) Research Institute for Intelligent Computer Systems, 2016. All rights reserved.
\end{abstract}

Keywords: Random Graphs, Small-World Networks, Optimization Strategies, Deterministic, Neuronal Networks.

\section{INTRODUCTION}

A small-world network is the notion that a short chain of intermediate acquaintances, which is characterized by a separation length of about six steps, can connect almost any pair of people in the world to one another. Small-world models for social networks display a large clustering coefficient; a high local clustering with disjoint regions that on average are connected to any node by only a few steps. A short summary of some background and models attempting to explain small-world phenomena are presented in $[2,24,27,30]$.

This paper presents an overview of small-world models, based on rules applied with small random probabilities. This approach makes sense if Nature is truly a stochastic process [7]. As a modeling tool, a random approach provides a high-level characterization of a complex behavior with minimal details of physical or systemic rules. However, it can also be argued that without specific details; random approaches may illustrate gross effects but, yet, obscure the causal details of resource and technology limitations and their economics of scale. This paper presents a different perspective in the understanding of small-world models by examining the design of a neuronal network simulator that is found to exhibits small-world features. In the remainder of this paper, we present the notion of six degrees of separation, current small-world models, and in particular the design of a neuronal-axon network simulator, its small-world organization, and the emergence of the small world from the application of optimization considerations.

\section{SIX DEGREES OF SEPARATION}

Six degrees of separation is the notion that everyone is a six or fewer step away from one another in the world by way of acquaintances. As a consequence, a chain of "a friend of a friend" inquiries can be made to connect any two people within a maximum of six steps. The original idea is by Frigyes Karinthy (a Hungarian author, playwright, poet, journalist, and translator). He was the first proponent of the six degrees of separation concept, where in his 1929 short story, Chain-Links, the author writes: " . . . to find a chain of contacts linking myself with an anonymous riveter at the Ford Motor Company - and I accomplished it in four steps. The worker knows his foreman, who knows Mr. Ford himself, who, in turn, is on good 
terms with the director general of the Hearst publishing empire [22]." The idea was further popularized in the 1990 play "Six Degrees of Separation" written by John Guare [12], which premiered on Off-Broadway. In this play, the character Ouisa asks "Six degrees of separation between me and everyone else on this planet. But to find the right six people." Building on this theme, Brian Turtle, Craig Fass and Mike Ginelli in 1994 created the game "Six Degrees of Kevin Bacon" where players try to connect any movie star, living or dead, to the veteran Hollywood actor Kevin Bacon. The game has become so successful that Google has incorporated the game into their search engine.

Surprisingly 25 years earlier, Casper Goffman described the idea of the Erdös number in his 1969 article entitled "And what is your Erdös number?" [11]. Paul Erdös was an influential mathematician who published more papers during his lifetime than any other mathematician in history. The idea of the Erdös number was originally created to describe the "collaborative distance" between the mathematician Paul Erdös and others who collaborated with him in authorship of mathematical papers. However, in later years the Erdös number has been generalized as an analogous notion of collaboration distance: two persons are linked if they are coauthors of an article.

\subsection{SMALL WORLD}

Milgram [23] provided one of the first studies in quantifying the small-world property of networks. His work explored the average number of steps that separated two individual from each other through a social network of friends-of-friends. The average separation is the number of steps that a message is passed between individuals, chosen from a random source, to arrive at a particular destination.

The results of the experiments show that the average number of steps needed is surprisingly small.

The small-world, social network relies on two properties: 1) on average a person's friend-of-afriend are far more likely to be acquainted with one another than two people chosen at random (clustering); and, 2) it is possible to connect two people chosen at random via a chain of only a few intermediate acquaintances (six degrees of separation).

A slightly different approach is to construct short paths based on local information. When network models are able to do this efficiently, the network is called navigable. Local information is defined as messages passed only between friends without knowing the exact path on subsequent passes. One strategy is to pass the message to the friend that appears closest to the destination (as measured in a social relational sense). Algorithmically, this approach implies a variant of a greedy routing strategy to find short paths in a network. This strategy forms the basis for a decentralized view that is used for navigation and searching in small-world models.

Small-world networks provide an important mathematical framework for modeling real-world social interactions. The spread of diseases [21, 26], a basis for epidemiological theory (random-mixing models) [17], social networking theory, and the design and analysis of the Internet infrastructure [1] are examples where the application of small-world networks has importance.

\subsection{RANDOM GRAPHS}

The theory of random graphs $[3,8]$ provides a framework where short paths can exist in large networks. Such a graph is denoted as $G(N, p)$, where $N$ is the number of nodes (vertices) and $p$ is a fixed probability of connecting an edge between a pair of vertices. Assume that $z$ is the number of edges per node (on average). Then the number of edges between nodes in the graph is $\frac{1}{2} \mathrm{Nz}$. Now select $N$ nodes and draw $\frac{1}{2} N z$ edges between randomly selected pairs, where each pair of nodes is connect by an edge with probability $p$. A graph with no edges has $p=0$, whereas, a fully connected graph has $p=1$.

To understand the small-world effects of random graphs, consider a node $N_{i}$ with $z$ neighbors. Assume that each of $N_{i}$ 's neighbors also has $z$ neighbors, which implies that $N_{i}$ has $z^{2}$ second neighbors. By extending this argument, the number $D$ degrees of separation needed to reach all $N$ nodes in the network is given by

$$
z^{D}=N \rightarrow D=\frac{\log N}{\log z}
$$

It is possible that the second neighbors of $N_{i}$ are also neighbors of $N_{i}$. In that case, these nodes form a clustering of the network. Unfortunately, the clustering of networks in a random graph is diminished by the likelihood that the selected pairs of nodes are connected with a probability $p$ in a uniformly random way.

The clustering coefficient $C$ is the average fraction of pairs of neighbors of a node that are also 
neighbors of each other. A fully connected network has a clustering coefficient $C=1$. The clustering coefficient for a random graph is $C=\frac{z}{N}$.

A measure for typical separation between vertices in a graph is denoted by $L(p)$ where $p$ measures the range of randomness for a graph, 0 (ordered) $<p<1$ (disordered) .

Table 1 illustrates an analysis of three different networks by Watts and Strogatz [31]. In the table, the corresponding $z$ values (not shown) are $z=2.67$ for the power grid (western USA), $z=14$ for the C. elegans and $z=61$ for the movie actors.

Table 1. Watts and Strogatz (1998)

\begin{tabular}{|c|c|c|c|c|c|}
\hline Network & N & Lactual & Lrand & Cactual & Crand \\
\hline C. elegans & 282 & 2.65 & 2.25 & 0.28 & 0.05 \\
\hline Power Grid & 4941 & 18.7 & 12.4 & 0.08 & 0.005 \\
\hline Film actor & 225,226 & 3.65 & 2.99 & 0.79 & 0.00027 \\
\hline
\end{tabular}

\section{BUILDING GRAPHS WITH BOTH SMALL-WORLD AND CLUSTERING PROPERTIES}

Random graphs show small-world properties but restrict the formation of clusters of vertices. Since clustering is an important property of real-world situations, a graphical network model has significance in the analysis and modeling of both physical and social networks [19]. In this section we will consider the models of Watts and Strogatz, and Kleinberg.

\subsection{WATTS-STROGATZ MODEL}

The model of Watts and Strogatz [31] is based on the assumption that people are more likely to have friends nearby, but still have some friends that live at a far distance. With these assumptions, the WattsStrogatz's small-world model exhibits the high clustering found in social networks.

The Watts-Strogatz graph is a one-dimensional, regular circular lattice (discrete, and with periodic boundary conditions) that allows a small degree of randomness to produce the small-world effect (see Fig. 1). Initially each node is assigned a position in the lattice and an edge is added between each node and its nearest neighbors to form the onedimensional lattice. Next a fixed (but small) number of edges are randomly rewired between nodes and may stretch long distances within the lattice.

The parameter, $p$, denotes the fraction of edges that are subject to random rewiring. The variation of $p$ makes possible the transition from a very locally ordered/clustered graph $(p=0)$ to one where all edges are randomly rewired $(p=1)$. The rewired edges create shortcuts that cover large distances in the underlying lattice. These shortcuts make possible the access, within a small number of steps, to vertices that are far away in the graph. Watts and Strogatz showed that the average path length between two nodes in a random network is equal to $\ln N / \ln K$, where $N=$ total nodes and $K=$ acquaintances per node. Thus if $N=300,000,000$ ( $90 \%$ of the US population) and $K=30$ then Degrees of Separation $=19.5 / 3.4=5.7$ and if $N=6,000,000,000 \quad(90 \%$ of the World population) and $K=30$ then Degrees of Separation $=22.5 / 3.4=6.6$.

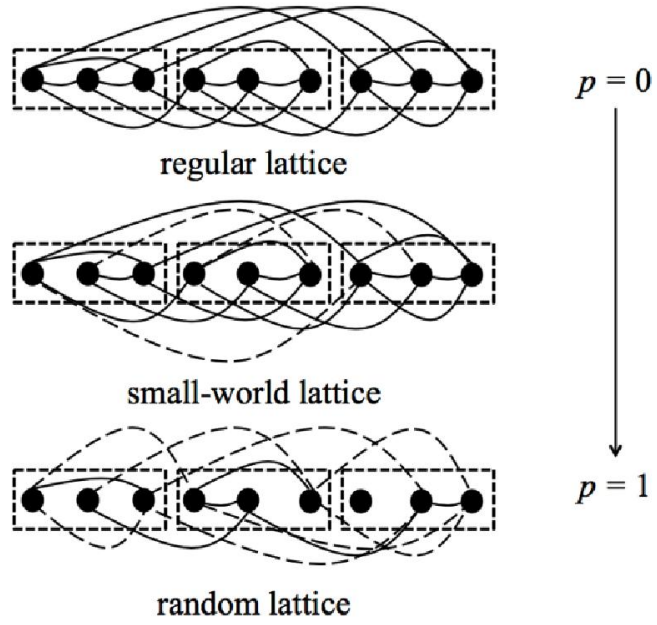

Fig. 1 - Lattices: Ordered, Small World, Random

The Watts-Strogatz model exhibits a rapid drop in the shortest path length and network diameter with only a small fraction of random rewiring. Albert and Barabási [2] provide additional details and simulations.

\subsection{KLEINBERG MODEL}

Kleinberg (2000) [18] generalizes the WattsStrogatz model and argues that models with shortcuts that are arbitrarily far apart are poor representations for some real-world situations. Kleinberg observations that people using local information are able to find short paths between individuals. He shows that the Watts-Strogatz model does not allow an efficient greedy routing algorithm to find short paths given only local information.

Kleinberg's model is a $k$-dimensional lattice with local connections to the nearest-neighbors. The probability for a shortcut edge to be added between two nodes is proportional to a distance measure $d(x, y)$ between each pair of nodes $x$ and $y$ that falls off as a power law proportional to $d(x, y)^{-p}$. Kleinberg shows that when $p=k$ an efficient greedy routing algorithm can be constructed to find short paths with only the use of local information. 
The corresponding probability for adding a shortcut edge from $x$ to $y$ is given by

$$
p(x \leftrightarrow y)=\frac{d(x, y)^{-k}}{H_{k}(n)}
$$

where $H_{k}(n)$ is a normalization constant.

The Kleinberg model adds shortcuts between nodes; whereas, the Watts-Strogatz model rewires existing edges in the lattice. For $p=0$, the Kleinberg model corresponds roughly to the WattsStrogatz model. As $p$ increases, the length of a shortcut edge becomes shorter with respect to the distance between nodes in the lattice. $k$ is the critical value for $p$ that couples the dimensionality of the lattice with the probability distribution needed to balance the shortcut distances in this greedy routing algorithm. An algorithm where shortcuts have been generated under this distribution takes an average complexity of $O\left(\log ^{2} n\right)$ steps. Newman and Watts [25] also considered the idea of adding shortcut edges in place of rewiring existing edges.

\section{A SMALL-WORLD BIOPHYSICAL NEURONAL-AXON NETWORK TOPOLOGY}

In this section, a small-world network topology of a distributed processing system is described. The architecture is designed as a parallel processing platform to simulate the synaptic response to the Hodgkin-Huxley (H-H) equations [14]. These equations model the activation and propagation of electric currents along the membrane of the squid giant axon. The axons are modeled as a long cylindrical tube, where electrical signals propagate along their outer membrane. The membrane is permeable to preferential chemical elements; namely potassium $(\mathrm{K}+)$ and sodium $(\mathrm{Na}+)$. The $\mathrm{H}-\mathrm{H}$ equations describe the sum of ion channels $(\mathrm{K}+$ and $\mathrm{Na}+)$ and the capacitance of the axon membrane.

\subsection{COMPUTATIONAL PARALLELISM}

An efficient parallel implementation of the $\mathrm{H}-\mathrm{H}$ model does not require a parallel decomposition of the equations. Instead, each neuron can be processedindependently from one another; therefore, a high degree of computational parallelism $\mathrm{O}(N)$ can be achieved, where $N$ is the number of neurons in atypical neuronal cluster. Consequently, the intrinsic parallelism would appear to be scalable and suitable for implementation on many low-end clustersystems, where an equal number of neurons are assigned to each of $P$ processors $(P<<N)$ to maintain computational work-load balance.

In designing an efficient parallel simulation platform, the computational parallelism, aggregatedata communications, and memory resources and organizationare fundamental criteria that need careful consideration. Too often the computational parallelism takes on the central focus of the implementation and relegates the importance of the ensuing data flow, and optimal data organization as secondary. As a consequence, concentrating exclusively on the extraction of the highest degree of computational parallelism can result in severely limiting the overall performance of the system platform.

\subsection{DATA-FLOW COMMUNICATIONS}

As for the data flow of the H-H model, when a neuron cell is stimulated above a given threshold, it transfers (communicates) that stimulus (an action potential) to all neuron cells connected to it. In a typical simulation, neurons are modeled as a completely connected cluster of approximately 10,000 neurons. As action potentials are transferred between each neuron, the aggregate of these neurons will also transfer their computed action potentials back to the first neuron. On a parallel system, the one-to-all and all-to-one communications patterns results in an all-to-all communications data transfer. In addition, the exchange of electric stimuli between connected neurons must involve all processors within a distributed architecture. Unfortunately, an all-to-all communications between processors lead to high communication latencies and a degradation in parallel performance.

A Beowulf computing system is one popular network topology that is deployed as a local area network. In such a network, Ethernet cables provide the connectivity that allow for "peer-to-peer" connections. For a Beowulf system, the Ethernet cabling imposes a bandwidth constraint on the multiple parallel transfers of action potentials among all attached processors. In addition, this limitation does not scale well as the number of processors, $P$, increase.

In a modern Ethernet, the processors do not all share a single channel through a shared cable; instead, each processor communicates through switches that in-turn forward data packets to the destination processor (see Fig. 2). In this topology, collisions are possible if processor and switch attempt to communicate with each other at the same time; however, this situation can be avoided in a full duplex mode of operation where switch and processor can communicate with each other simultaneously. In full duplex mode, when one 
processor sends data packets to all other processors (broadcast or one-to-all communication), the Ethernet-switch topology is very efficient; assuming that there are enough switch nodes available to service all the data traffic demands. However, when all processors attempt to send packets simultaneously in a one-to-all communications pattern, packet collisions cannot be avoided. As a consequence, choosing an appropriate network topology for the desired simulator is an important design consideration and one that requires an understanding of network latencies and resource limitations.

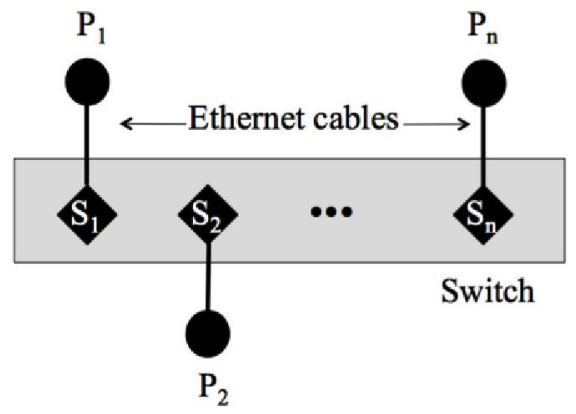

Fig. 2 - Beowulf Ethernet-Switch Topology

As a side note, the notion of scaling refers to the process of increasing the problem size in proportion to an increase in the number of additional processors added to the system. The H-H model follows a simple memory-scaling rule that grows linearly in the number of action potential tokens generated and their subsequent transfer across processors. Therefore, for $Q$ additional processors added to a $P$ parallel processor system, the memory required per processor is given by $N / P$ where $N$ is the total number of simulated neurons.

If $N$ grows proportionally with $P$, then

$$
N / P=N^{\prime} / P^{\prime}
$$

or

$$
N^{\prime}=(N / P) \times(P+Q)
$$

where $P^{\prime}=(P+Q)$.

Eqn. 1 represents not only the maximum system memory but also the maximum number of action data-flow tokens that need to be transferred throughout the network (minus the tokens that are communicated to neurons within each processor).

An alternative choice is a simple star network as illustrated in Fig. 3. This configuration provides a convenient clustered topology where the central node is one hop away from every other node. In order to support an all-to-all communications capability, a logical extension might be a completely connected star network as illustrated in Fig. 4. The completely connected star network retains the one hop property that links any two processing nodes. This property guarantees a highly cluster network topology where data transfers would appear to be optimal. Unfortunately, this network topology is not scalable, because as the number of processors increase the number of communication links grows as $\mathrm{O}\left(P^{2}\right)$.

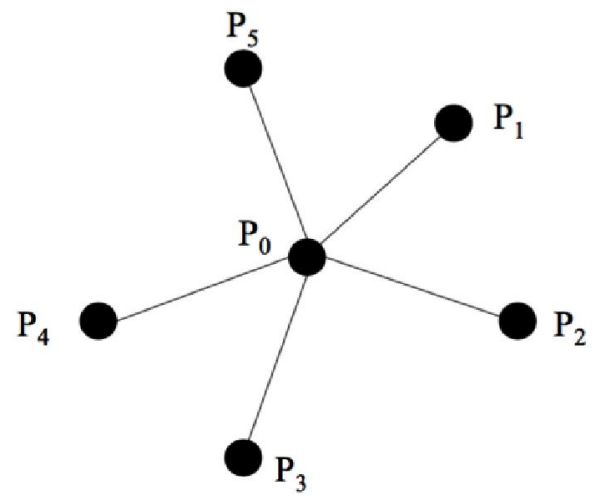

Fig. 3 - Star Network

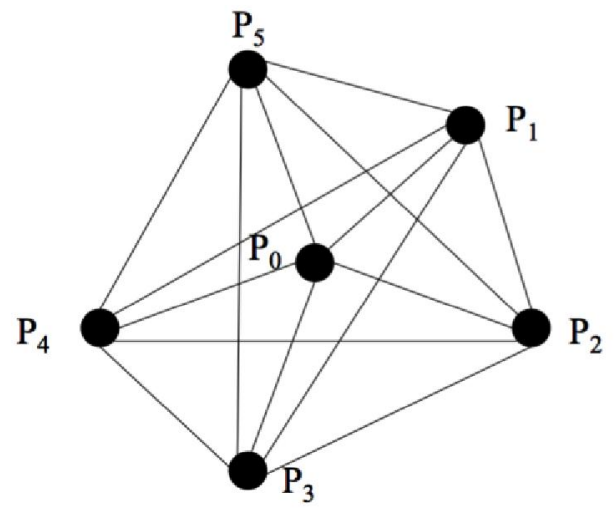

Fig. 4 - Completely Connected Star Network

A rearrangement of the star network readily reveals a more familiar Master/Slave topology (Fig. 5), where the number of network connections grows linearly in the number of Slave processors $(P)$. This topology is simple, scalable in processor utilization and requires minimal communication channels.

However, three potential drawbacks limit the efficiency of this particular processor-network topology. First, most commodity processors do not support parallel I/O when distributed data packets are sent to or from a single processing node. Instead the CPU or most likely a coprocessor provides the communications capability. This arrangement results in serializing communications with a timecomplexity that grows as $O(P)$. 


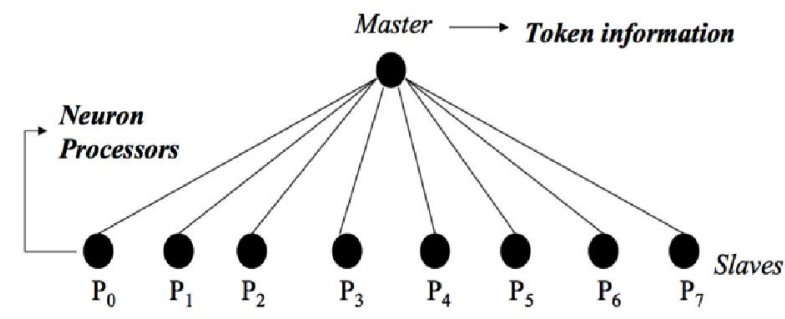

Fig. 5 - Master/Slave Topology

Second, when multiple nodes simultaneously send packets to a single destination node, an all-toone synchronized communications scheme is required to avoid packet collisions on the network. Third, the memory capacity of the Master node is sensitive to problem-size scaling according to Eqn. 1. This is in contrast to the Slave (Neuron) nodes that maintain a constant memory size of $N^{\prime} / P^{\prime}$. In this sense, the neuron-axon problem is very scalable with regards to the neuron processor parallelism. However, the Master node, which plays the key role in managing the receiving and sending of all actionpotential tokens between processors, becomes a memory bottleneck as the total problem size increases. If $T_{a p}$ is the number of action-potential tokens generated per processor, then the Master node memory capacity, $M_{c}$, must grow as a function of the number of processors and actionpotential data tokens transferred:

$$
M_{c}=\sum_{i=1}^{P}\left(T_{a p}\right)_{i}
$$

which also defines the limiting scalable problem size and the degree of parallelism that can be simulated with a fixed Master node memory capacity.

A simpler network topology is a ring network, where all action potentials are transferred along a single direction (see Fig. 6). For this topology, action potentials are removed as they arrive at their destination processor; whereas, the remaining action potentials are transferred further along the ring. In this situation, the degree of communications parallelism is $O(P)$; however, the number of communication hops required to complete a communications cycle also grows as $O(P)$. On the other hand, the advantage of the ring network is that for a fixed channel bandwidth the number of action potentials transferred depends only on the number of neurons per processor, $N / P$. In addition, the scaling of the problem size with number of processors does not require an increase in the memory size for all ring node processors. This is in contrast to the Master/Slave scaling characteristic.

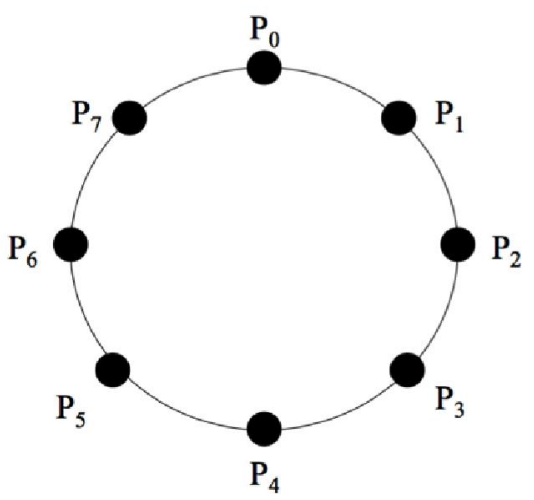

Fig. 6 - Ring Network

An application of small-world transformations, by either reconnecting a few edges (links) (Fig. 7(a)) or adding a few additional edges (Fig. 7(b)), does not enhance the optimal ring communications routing algorithm. In Fig. 7(a), the reconnection of links creates a broken ring topology that disrupts and degrades the simple and efficient ring data routing protocol.

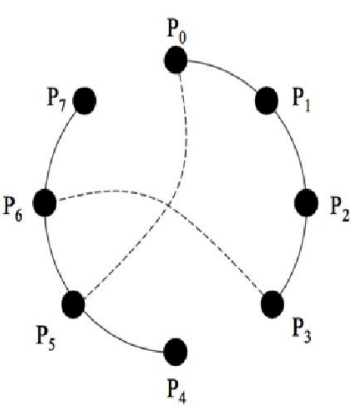

(a)

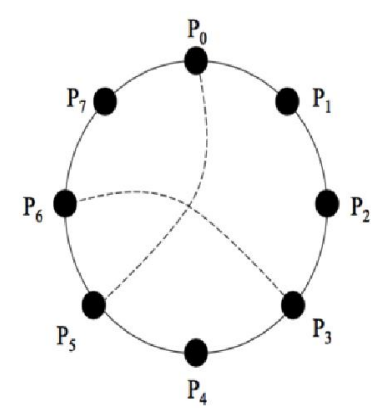

(b)
Fig. 7 - Small-World Ring Network

On the other hand, the addition of several edges (Fig. 7(b)) increases (enriches) the ring connectivity per node but does not provide any useful advantages to improve the intrinsic optimal ring routing algorithm.

A final architectural consideration is a tree topology where the interior nodes are replaced with simple router switches to manage the data-flow traffic. Fig. 8 illustrates the proposed architecture as a balanced binary tree, although this is not necessary. The architecture increases in the number of processors by $P=2^{i}$ where $i$ is the height of the tree, and the ratio of switches to processors remains constant. The tree-structured topology is organized into three distinct regions: 1) the root node that bridges the left- and right-subtrees, 2) the leaf-nodes, $P_{i}$, that make up the neuron processors, and 3) the interior nodes of the tree that manages the datatoken traffic through the various router switches. 


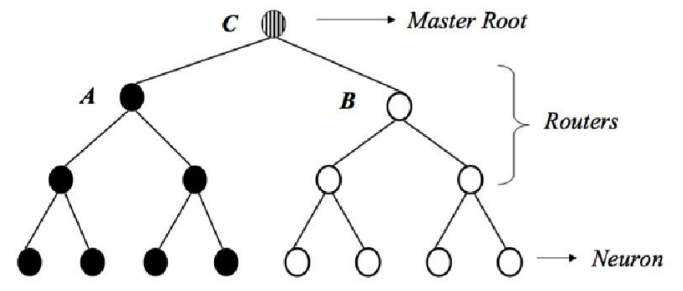

Fig. 8 - Binary Tree Topology

The routers sort the received action-potential tokens into two data token groups: those tokens that are sent up the tree towards the root node, and those tokens that are sent down to their respective left- and right-subtrees, depending upon the tokens' designated destinations. This routing enhancement avoids the transfer of all tokens through the root node. As a consequence, a significant reduction in the required memory capacity of the root node is achieved as well. When compared to the Master/Slave topology described above, the tree topology exhibits a more optimal simulation platform where: 1) data-flow routes can be performed in parallel, and 2) the farthest neuron processors can be reached in $O(\log P)$ steps, and 3 ) the minimal working root node memory capacity is reduced by a factor of

$$
\frac{P}{2(P-1)} \approx \frac{1}{2}
$$

Replacing each router switch with a processor that acts as both a neuron processor and a router can also modify the proposed tree topology. In effect, this modification would not change the network's communications complexity; while doubling the computational parallelism by a factor of 2 . In such a configuration, a larger simulation problem can be implemented. In either case, the maximum distance (hops) between nodes in the network is given by a distance complexity of $O(\log P)$.

It is interesting to note that the $O(\log P)$ complexity is a property of many optimal algorithms designed for both sequential and parallel problems. This distance complexity is also a characteristic encountered in the Watts-Strogatz small-world model, and introduced to optimize the clustering between long-distant neighborhoods. In a similar fashion, the proposed tree-structured neuronal-axon simulation platform is designed to achieve the same $O(\log P)$ neighborhood accessibility as described by the Watts-Strogatz model.

\subsection{MEMORY LIMITATIONS}

Although novice computer practitioners frequently quote the mantra "memory is cheap," memory is a key enabling resource that requires serious consideration and analysis. The memory reduction (Eqn. 2) of the root node of the tree topology in comparison to the Master node of the Master/Slave configuration, points to the importance of analyzing the memory complexity of various architectures. This is illustrated when a computational task exhausts the available memory capacity; the execution halts and no further progress can be made. This termination occurs even if the software is error free.

The tree topology offers routing opportunities that result from paired links between a parent and its corresponding children nodes. As a consequence, these links provide additional routes that avoid the need to communicate through the root node; such that, the root node memory size is reduced by a factor of two as compared to the Master node in the Master/Slave network topology. Using these ideas, the notion of a bisected-binary-tree (BBT) architecture is introduced that provides a convenient mechanism to further reduce the memory utilization. The BBT is illustrated in Fig. 9.

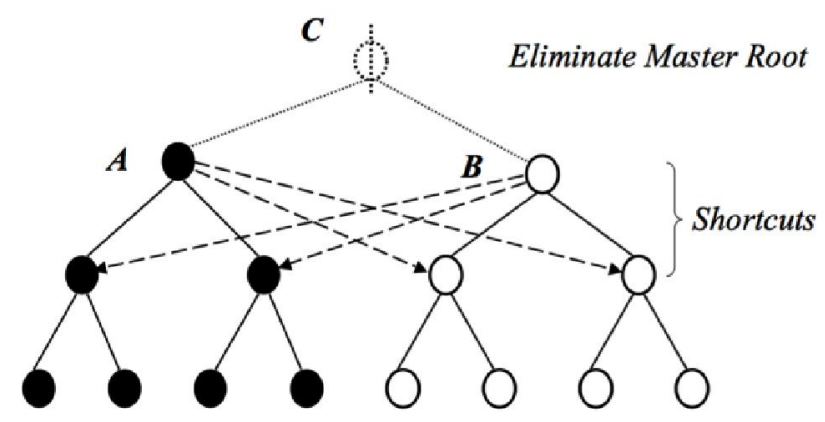

Fig. 9 - Bisected-Binary Tree Topology

The BBT is a modification of a binary tree, where the root node $(C)$ is removed (i.e., bisect the binary tree) and shortcut links (indicated by the dashed arrows) are added to the two routing nodes labeled $A$ and $B$. In this construction, the left-subtree root node $(A)$ connects directly to the right-subtree of $B$, and the right-subtree root node $(B)$ connects directly to the left-subtree of $A$. This bisection process incorporates a rewiring of existing edges that are connected to node $C$, and the addition of new shortcut edges from nodes $A$ and $B$ to their corresponding right- and left-subtrees, respectively. Although the BBT increases the connection complexity, it also increases the clustering for specific nodes in a non-random way. In this example, the BBT is referred to as a level-1 BBT. The application of the BBT method is not restricted to the level-1 BBT. In fact the BBT method can be applied recursively to create multiple levels of bisection as seen in Fig. 10. 


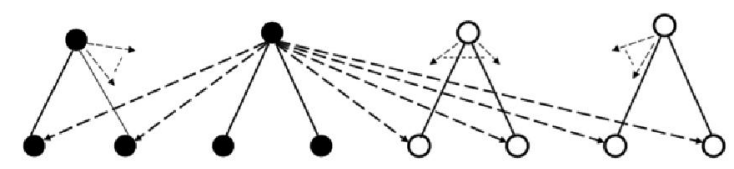

Fig. 10 - Level-2 Bisection

In general, the level- $i$ BBT has $R_{i}$ sub-router root nodes with $L_{i}$ additional links that connect to routers or neuron processing nodes at the (i+1) level. The number of sub-router root nodes and the additional links is given by

$$
\text { Level-i } \sim\left\{\begin{array}{c}
R_{i}=2^{i} \\
L_{i}=2 \times\left(2^{i}-1\right)
\end{array}\right.
$$

where $i=0,1, \ldots, \mathrm{n}$.

The BBT construction results in small-world clustering properties and a characteristic path length $O(\log P)$. The approach increases efficient memory utilization and induces a welcomed clustering of nodes within the network, but at the expense of introducing long-distance shortcuts and the addition of $2^{i+1}-2$ links to each sub-router root node. The Watts-Strogatz model also suffers from the introduction of long-distance shortcuts. Kleinberg's model avoids long-distance shortcuts using of a power law distribution model. However, the longdistance shortcuts can be avoided by introducing a higher-dimensional hierarchical organization that accomplishes the same goal. Tanay et al., [28] give evidence of such a hierarchical organization in the yeast molecular network. Fig. 11 illustrates how the added dimensionality can re-scale long-distance shortcuts by re-arranging their orientation.

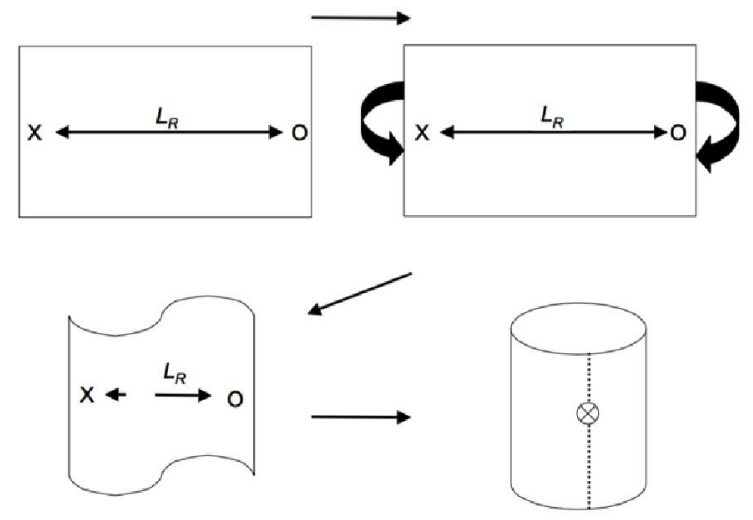

Fig. 11 - 3-Dimensional Space

The bisected tree graph with shortcuts can be reconfigured in a three-dimensional space by first applying a two-dimensional rotation on one of the sub-trees (see Fig. 12) followed by an overlay using the third dimension. Fig. 13 illustrates the final network topology that is free of long-distance shortcuts.
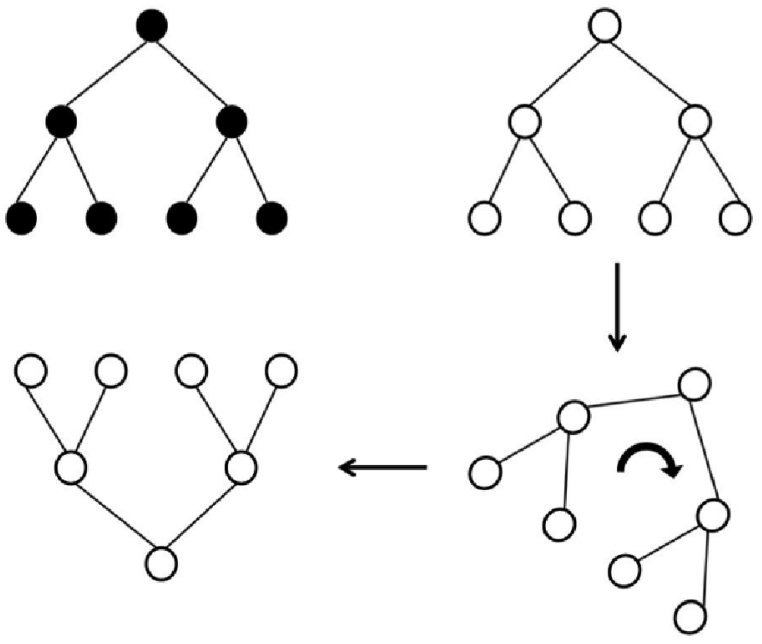

Fig. 12 - Two-Dimensional Rotation

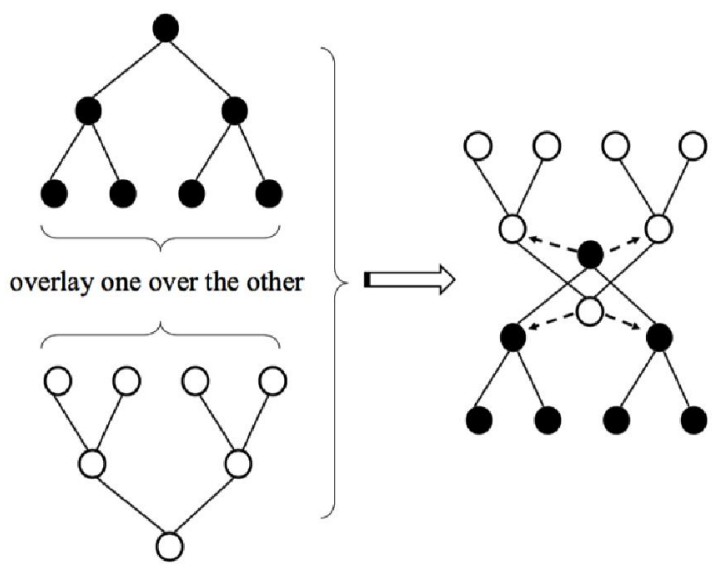

Fig. 13 - Overlay in Third Dimension with Added Shortcuts

One troubling aspect of the BBT construction is the addition of $2^{i+1}-2$ shortcut links to each subrouter root node. As $i$ increase, the network performance incurs a noticeable communications delay as the sequential I/O capacity of each subrouter root node becomes apparent. Therefore, the BBT is limited in the number of recursive applications for performance and practical reasons. This is also a physical limitation shared by all network configurations. Fig. 14 illustrates a fan-out of shortcuts. In integrated circuits, the fan-out of $N$ logic gates connected to the output of a driving gate can experience propagation and timing delays, and false logic ( 1 or 0$)$ signals [33]. In the later case, for any driving gate there exists a maximum value of the current, $\mathrm{I}_{\max }$, which can be divided among $\mathrm{N}$ receiving gates. If each receiving fan-out gate operates at a current level greater than $\mathrm{I}_{\text {in }}=\mathrm{I}_{\max } / N$, then distorted (incorrect) logic signal are received. 


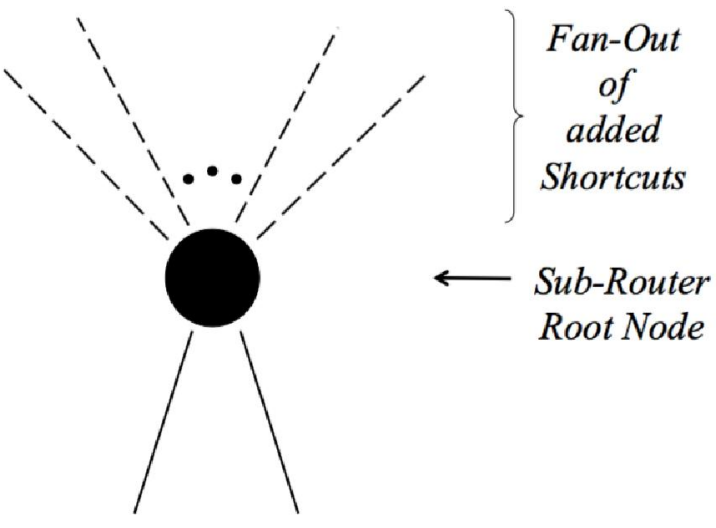

Fig. 14 - Shortcuts

Large networks are all subject to distributed networking substructures to maintain efficient and reliable performance. A cellular phone system is an example of a large network that is maintained through a large number of access points and base stations that coordinate the use and movement of users within the wireless network.

An analogous example is the structure of the neuron itself. Consider a simple model of an accumulated action potential strength applied to a typical neuron. Fig. 15 illustrates a typical multipolar neuron that has three components: cell body, dendrites and an axon. The cell body contains the nucleus and bears-short branched filaments called dendrites that transmit impulses from synapses to the cell body of other neurons. The axon is an elongated tapered strand, which carries an action potential impulse away from the cell body across synapses to other neurons.

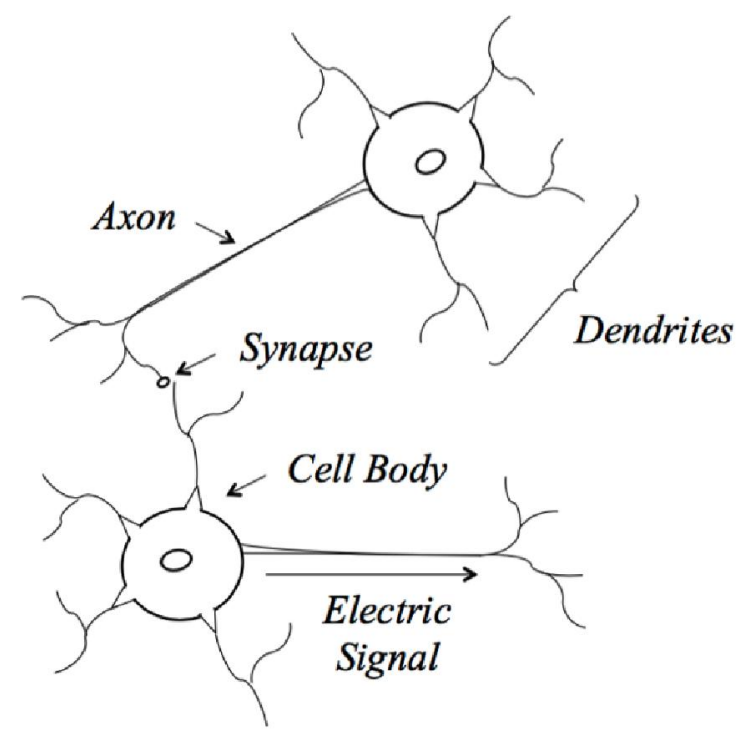

Fig. 15 - Biological Multipole Neuron

If we assume that one neuron can stimulate a large number of neighboring neuron cells (e.g., $10,000)$, then it is also possible that one neuron can be stimulated by all neighboring neurons. Suppose that the aggregate of action potentials $A_{N}$ from all $N$ contributing neurons arrive at a single neuron at one instant in time, and its influence is given by a Big- $\Theta$ complexity of

$$
A_{N}=\Theta\left(\sum_{i=1}^{N} \Delta_{i}\right)
$$

where $\Delta_{i}$ is the individual action potential sent by the $i^{\text {th }}$ neuron.

Now suppose that $A_{\max }$ is the maximum stimulus strength that a single neuron can receive without being damaged. If

$$
\Delta_{i} \propto \frac{A_{\max }}{M},
$$

and if $M<N$, then it is possible that the receiving neuron cell body could experience a combined stimulus for which

$$
A_{N}>A_{\max }
$$

The resulting activation can cause cell damage or at least an overly saturated (stressed) neuronal state. This situation can be avoided by organizing neurons into a clustered network with small-world degrees of separation, rather than a prohibitively large, completely connected network as illustrated in Fig. 4.

Of course, the network organization, argued for here, is one of several mechanisms that can limit or avoid neuronal saturation. The number of dendritic inputs to a given neuron as illustrated in Fig. 15 is one such mechanism. Another mechanism is the synaptic signals from other neurons that may trigger excitatory or inhibitory modes of potentiation. The combination of these triggered modes can provide a throttling mechanism to manage activation pulse levels.

\section{A SMALL-WORLD FOR A NEUROANATOMY MODEL}

A. Damasio [5] proposed a neuroanatomy model based on experimental evidence to address the binding problem: the integration of both the sensory and motor components in both recognition (perception) and recall. The properties of objects and events that are perceived through the various sensory interactions rely on geographically separate sensory regions of the brain.

He found no structural evidence to support the intuition that temporal and spatial integration occur 
at a single site. He maintains that the integration of multiple aspects of reality, external and internal, links together distributed repository of fragmented encoded sensory information; stored in remote and geographically separate locations within sensory and motor regions, and reconstructed by co-activation zones. He proposed a neuroanatomical network that allows for both forward propagation and convergence of parallel streams of sensory data with backward propagation of signals back to the points of origin.

The representations of objects with spatial and temporal associations are stored in separate neural regions called convergence zones. The reactivation of recall requires the firing of convergence zones with feedback streams propagating from them. Convergence zones bind neural activity patterns corresponding to topographically organized fragments. The geographic location of convergence zones varies among individuals but is not random.

Fig. 16 is an illustration of a diagram appearing in [6]. The described neuroanatomy network can be viewed as a multi-layered tree topology, where internal sensor and motor components are represented at one level and a combinatorial layer is depicted at a higher level. Small-world shortcuts can be viewed as paths for forward and backward data propagation, where re-enforcement learning or sensitivity calibration can be performed. These shortcuts involve both local routing operations internal to each sensor and motor components, and global routing operations among all components.

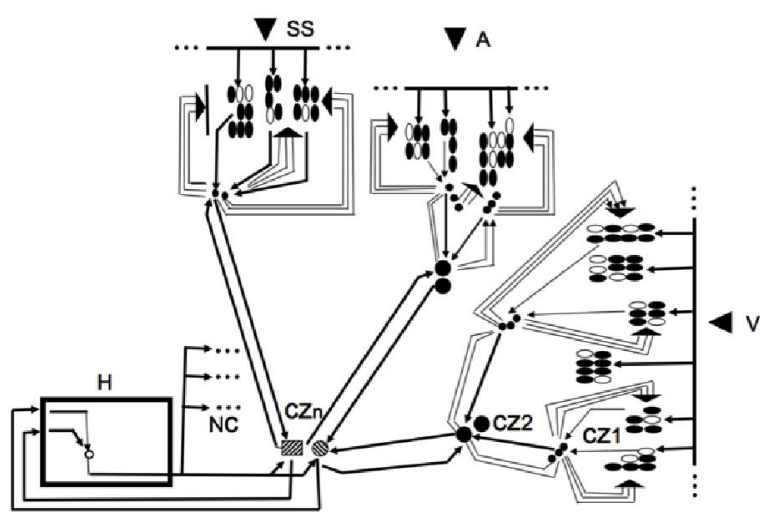

Fig. 16-Neuroanatomical network

The neural architecture depicts the integration of visual (V), somatosensory (SS), and the auditory (A) sensor regions of the cortex. The filled and unfilled dots represent separate functional regions in each of the sensory areas. The arrows pointing from the sensory regions to the convergence zones ( $\mathrm{CZ1}$, $\mathrm{CZ2}, \mathrm{CZn}$ ) represent feed-forward paths. Arrows pointing back to the sensory regions represent feedback paths from each CZ. H depicts the hippocampal system. The outputs of $\mathrm{H}$ are returned to $\mathrm{CZn}$ and to the non-cortical neural locations of the basal forebrain, brain stem, and the neurotransmitter nuclei. The feed-forward and feedback pathways terminate within the sensory regions over the aggregate of neurons in a distributed fashion, rather than on a specific neuron.

\section{CONCLUSION}

The small-world model is an organizational abstraction to provide insight into real-world phenomena exhibited by social, biological and manmade systems. The small-world models reviewed in this paper rely upon random graph techniques but applied within a constraint framework. These constraints represent rules that allow a small range of random flexibility to evolve into surprisingly rich organizational structures.

Unfortunately, the small-world abstraction is not intended to capture the physically criteria for these rules. Instead, small-world models seek rules that create clustering of vertices where the average distance between clusters are minimized by the introduction of shortcut edges. This high-level network modeling approach has been applied in behavioral modeling of epidemiological and social systems. However, deriving the rules for smallworld network clustering and connectivity rely on insights from observational experience.

In this paper, we explore the possibility of a small-world analogy that arises in the design of a neuronal-axon network simulator. The approach does not rely on random graph connections or reconnections. Instead the design considerations rely on exploring the minimal (optimal) operational complexity to achieve a reasonably robust simulation platform. Three key factors are used to guide the architectural organization of the final design. These factors are 1) the growth in the number of communication links per processor as the simulation size and number of processors is scaled up, 2) an efficient utilization of available memory, and 3) a characteristic path length $L$ between clusters that grows as $O\left(\log _{2} P\right)$.

Based on the three considerations listed above, the final network design is organized as a treestructured network. As with other described smallworld constructions, shortcut links are introduced into the resulting network topology design. However, the shortcuts presented here are introduced to reduce the demands on memory utilization as imposed on the root or sub-tree root nodes. These nodes in particular are involved in the action-potential-token traffic management among all neuron processors within the tree. So surprisingly, these shortcuts are not only distance minimizing but also memory minimizing. 
The network is reminiscent of both the WattsStrogatz and Kleinberg models, where the introduction of long-distance shortcuts are accomplished by a rewiring of existing edges and the addition of new edges. This combination of shortcuts occurs during the bisection process. On the other hand, the Kleinberg power-law distribution model for clustering is avoided since introducing shortcut connections in this manner would create data-flow traffic collision within the network topology (see Fig. 14). Instead, rotation and translation (overlay) operations are applied within a three-dimensional framework to reduce the lengths of long-distance shortcuts.

The binary-tree topology provides a convenient characteristic path length of $O\left(\log _{2} P\right)$. A 2-ary ncube (hypercube) topology also exhibits the same characteristic path length; however, the number of edges per hypercube node grows linearly with the dimensionality of the hypercube. For that reason, the hypercube was not considered.

Finally, it is shown how small-world phenomena arise from optimization constraints that place a balance on minimizing system complexities. In addition, the described approach avoids the introduction of random uncertainties to achieve certain modeling goals.

\section{REFERENCES}

[1] R. Albert, H. Jeong, A.-L. Barabási, "The diameter of the World Wide Web," Nature, vol. 401, issue 9, pp. 130-131, 1999.

[2] R. Albert and A-L. Barabási, "Statistical mechanics of complex networks," Reviews of Modern Physics, vol. 74, pp. 47-97, 2002.

[3] B. Bollobás, Random Graphs, Academic Press, London, 1985.

[4] I. Clarke, O. Sandberg, B. Wiley, and T. W. Hong, "Freenet: A distributed anonymous information storage and retrieval system," in Proceedings of the International Workshop on Design Issues in Anonymity and Unobservability, 2001, pp. 46-66.

[5] A. R. Damasio, "Time-locked multiregional retroactivation: A system-level proposal for the neural substrates of recall and recognition," Cognition, vol. 33, issue 1-2, pp. 25-62, 1989.

[6] A. R. Damasio, "The brain binds entities and events by multiregional activation from convergence zones," MIT Press, vol. 1, issue 1, pp. 123-132, 1989.

[7] M. Denny and S. Gaines, Chance in Biology: Using Probability to Explore Nature, Princeton University Press, 2000.

[8] P. Erdős and A. Rényi, "On random graphs," Publicationes Mathematicae, vol. 6, pp. 290297, 1959.
[9] M. R. Garey, D. S. Johnson, and L. Stockmeyer, "Some simplified np-complete problems," Theory of Computer Science, vol. 1, pp. 237-267, 1978.

[10] W. R. Gilks, S. Richardson, and D. Spiegelhalter, Markov Chain Monte Carlo in practice, Chapman and Hall/CRC, 1996.

[11] C. Goffman, "And what is your Erdös number?" American Mathematical Monthly, vol. 76, issue 7, p. 791, 1969.

[12] J. Guare, Six Degrees of Separation: A Play, Vintage Books, New York, 1990.

[13] O. Häggstrőm, Finite Markov Chains and Algorithmic Applications, Cambridge University Press, 2002.

[14] A. L. Hodgkin and A. F. Huxley, "A quantitative description of membrane current and its application to conduction and excitation in nerve," J. Physiol. (Lond.), vol. 117, pp. 500544, 1952.

[15] J. Kaiser, Ed., "It's a small Web after all," Science, vol. 285, p. 1815, 1999.

[16] H. Kautz, B. Selman, M. Shah, "Referral Web: combining social networks and collaborative filtering," Communications of the ACM, vol. 30, issue 3, pp. 63-65, March 1997.

[17] M. J. Keeling and K. T. D. Eames, "Networks and epidemic models," J. R. Soc. Interface, vol. 2, pp. 295-307, 2005.

[18] J. Kleinberg, "The small-world phenomenon: An algorithmic perspective," in Proceedings of the 32nd ACM Symposium on Theory of Computing, 2000.

[19] D. Knoke and S. Yang, Social Network Analysis (Quantitative Applications in the Social Sciences), Sage Publications, Inc.; 2nd Edition, November 2007.

[20] C. Korte and S. Milgram, "Acquaintance networks between racial groups: Application of the small world method," J. Personality and Social Psych., vol. 15, pp. 101-108, 1978.

[21] M. Kretschmar and M. Morris, "Measures of concurrency in networks and the spread of infectious disease," Mathematical Biosciences, vol. 133, pp. 165-195, 1996.

[22] A. Makkai and E. Jankó, Translated from Hungarian and annotated.

[23] S. Milgram, "The small world problem," Psychology Today, vol. 1, pp. 61-67, 1967.

[24] M. E. Newman, "Models of the Small World," J. Statistical Physics, vol. 101, no. 3-4, pp. 819841, 2000.

[25] M. E. Newman and D. J. Watts, "Renormalization group analysis of the smallworld network model," Physical Review Letters $A$, vol. 263, pp. 341-346, 1999. 
[26] M. E. Newman and D. J. Watts, "Scaling and percolation in the small-world network model," Physical Review E, vol. 60, pp. 7332-7342, 1999.

[27] O. Sandberg, "Distributed routing in smallworld networks," in Proceedings of the 8th Workshop on Algorithm Engineering and Experiments (ALENEX06), 2006.

[28] A. Tanay, R. Sharan, M. Kupiec, and R. Shamir, "Revealing modularity and organization in the yeast molecular network by integrated analysis of highly heterogeneous genome wide data," Proceedings of National Academy of Science USA, vol. 101, issue 9, pp. 2981-2986, 2004.

[29] J. Travers and S. Milgram, "An experimental study of the small world problem," Sociometry, vol. 32 , p. $425,1969$.

[30] D. Watts, Small Worlds, Princeton University Press, 1999.

[31] D. Watts and S. Strogatz, "Collective dynamics of small world networks," Nature, vol. 393, pp. 440-442, 1998.

[32] D. Watts, Six Degrees: The Science of a Connected Age, W.W. Norton \& Co., 2003.
[33] L. Berman, L. Carter, K. F. Day, "The fanout problem: from theory to practice," Yorktown Height, N.Y.: IBM T.J. Watson Research Center, 1988.

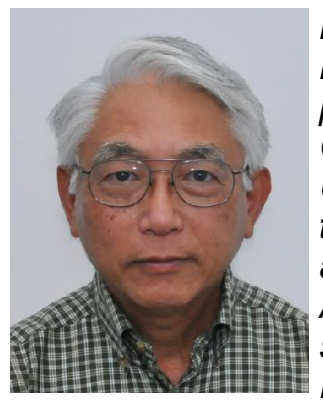

Robert E. Hiromoto is a Fulbright Scholar and a full professor in the Department of Computer Science at the University of Idaho, located in the Idaho Falls Center. He is affiliated with the Center for Advanced Energy Studies and supervises students from the Idaho National Laboratory. He holds a doctorate in physics and has done considerable work in parallel computational methods. His scientific research interests span the areas of distributive systems, parallel pseudorandom number generation, wireless communications protocols, and advanced algorithm design and optimization techniques for computational nuclear engineering problems. 